Yingying Han, Sisi Feng* and Liping Lu

\title{
Crystal structure of catena-poly[ $\left[\mu_{2}-3-\right.$ carboxy- 2,3-bis((4-methylbenzoyl)oxy)propanoato- $\mathrm{K}^{2} \mathrm{O}: \mathrm{O}^{\prime}$ \}tris(methanol-k ${ }^{1} \mathrm{O}$ ) lanthanum(III)], $\mathrm{C}_{63} \mathrm{H}_{63} \mathrm{LaO}_{27}$
}

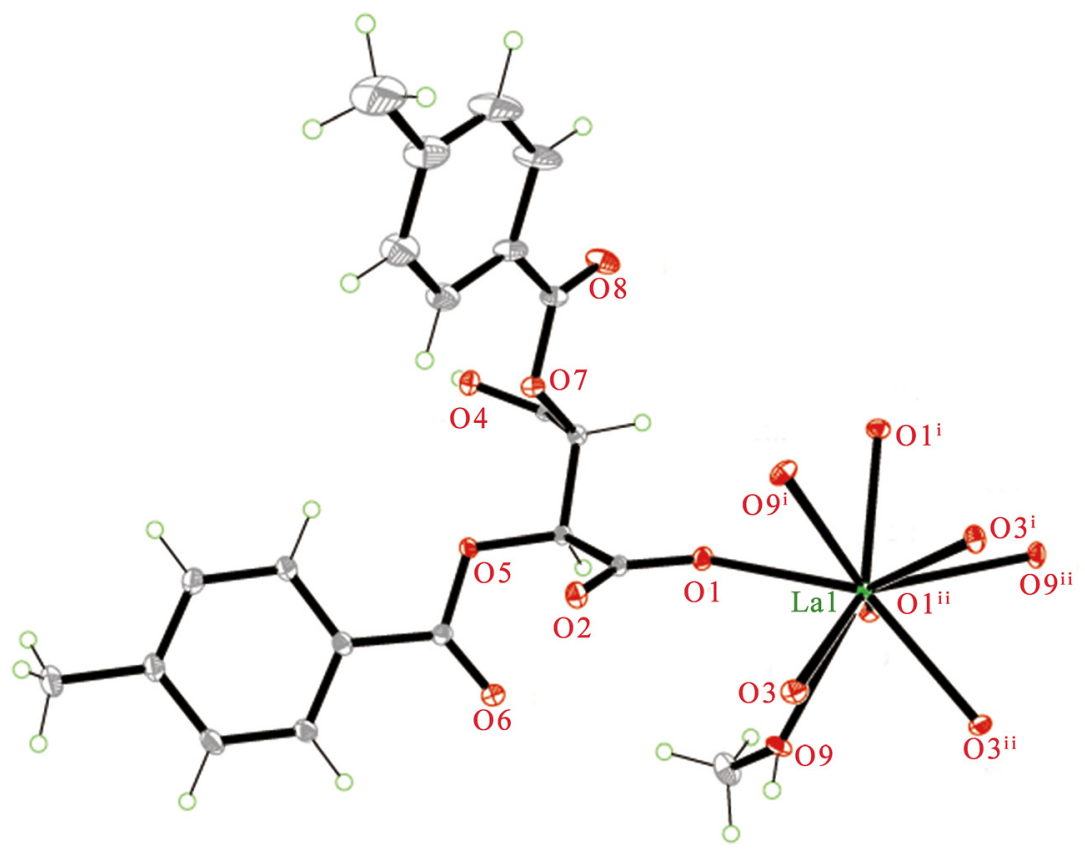

https://doi.org/10.1515/ncrs-2022-0022

Received January 16, 2022; accepted February 7, 2022; published online February 23, 2022

\section{Abstract}

$\mathrm{C}_{63} \mathrm{H}_{63} \mathrm{LaO}_{27}$, trigonal, $R 3$ (no. 146), $a=27.5427(11) \AA$, $c=7.7330(4) \AA, V=5080.3(5) \AA^{3}, Z=3, R_{g t}(F)=0.037$, $w R_{\text {ref }}\left(F^{2}\right)=0.096, T=100 \mathrm{~K}$.

CCDC no.: 2141982

A part of the title crystal structure is shown in the figure. Table 1 contains crystallographic data and Table 2 contains

*Corresponding author: Sisi Feng, Institute of Molecular Science, Key Laboratory of Chemical Biology and Molecular Engineering of the Education Ministry, Shanxi University, Taiyuan, Shanxi 030006, People's Republic of China, E-mail: ssfeng@sxu.edu.cn. https://orcid.org/0000-0003-2357-2995

Yingying Han and Liping Lu, Institute of Molecular Science, Key Laboratory of Chemical Biology and Molecular Engineering of the Education Ministry, Shanxi University, Taiyuan, Shanxi 030006, People's Republic of China
Table 1: Data collection and handling.

\begin{tabular}{ll}
\hline Crystal: & Colorless block \\
Size: & $0.30 \times 0.20 \times 0.20 \mathrm{~mm}$ \\
Wavelength: & Synchrotron radiation $(0.710 \AA)$ \\
$\mu:$ & $0.70 \mathrm{~mm}^{-1}$ \\
Diffractometer, scan mode: & mar555 \\
$\theta_{\text {max }}$, completeness: & $30.5^{\circ}, 99 \%$ \\
$N(h k l)_{\text {measured }}, N(h k l)_{\text {unique }}, R_{\text {int }}:$ & $9418,9418,0.000$ \\
Criterion for $I_{\text {obs }}, N(h k l)_{\text {gt: }}:$ & $I_{\text {obs }}>2 \sigma\left(I_{\text {obs }}\right), 9415$ \\
$N(\text { param })_{\text {refined: }}$ & 279 \\
Programs: & Data processing [1], \\
& Bruker [2], SHELX [3, 4] \\
\hline
\end{tabular}

the list of the atoms including atomic coordinates and displacement parameters.

\section{Source of material}

All reagents and solvents are analytically pure and used without further purification. The D- $\mathrm{H}_{2}$ DTTA (Systematic 
Table 2: Fractional atomic coordinates and isotropic or equivalent isotropic displacement parameters $\left(\AA^{2}\right)$.

\begin{tabular}{|c|c|c|c|c|}
\hline Atom & $x$ & $y$ & $z$ & $U_{\text {iso }}{ }^{*} / U_{\text {eq }}$ \\
\hline La1 & 0.666667 & 0.333333 & $-0.08427(2)$ & $0.01006(8)$ \\
\hline 01 & $0.71133(13)$ & 0.40985 (13) & $0.1242(5)$ & $0.0173(6)$ \\
\hline 02 & $0.75601(13)$ & $0.50115(13)$ & $0.0516(4)$ & $0.0180(6)$ \\
\hline 03 & 0.71745 (13) & 0.40974 (13) & $-0.3031(5)$ & $0.0174(6)$ \\
\hline 04 & 0.73019 (14) & 0.49588 (13) & $0.7414(4)$ & $0.0177(6)$ \\
\hline H4 & 0.739982 & 0.491713 & 0.840649 & $0.026^{*}$ \\
\hline 05 & $0.79391(12)$ & $0.53204(12)$ & $0.3805(4)$ & $0.0149(5)$ \\
\hline 06 & $0.86356(14)$ & $0.52462(15)$ & $0.2513(5)$ & $0.0243(7)$ \\
\hline 07 & $0.68455(12)$ & $0.49212(12)$ & $0.4194(4)$ & $0.0153(5)$ \\
\hline 08 & $0.60451(15)$ & $0.43367(17)$ & $0.5607(6)$ & $0.0281(8)$ \\
\hline 09 & $0.77891(12)$ & 0.37914 (14) & $-0.0984(5)$ & $0.0186(6)$ \\
\hline H9A & 0.794461 & 0.396249 & -0.193391 & $0.028^{\star}$ \\
\hline C1 & 0.74035 (16) & $0.46078(16)$ & $0.1547(6)$ & $0.0138(7)$ \\
\hline$C 2$ & $0.75774(16)$ & $0.47381(16)$ & $0.3473(6)$ & $0.0130(6)$ \\
\hline $\mathrm{H} 2$ & 0.776879 & 0.452735 & 0.383763 & $0.016^{\star}$ \\
\hline C3 & $0.70500(16)$ & $0.45470(16)$ & $0.4578(6)$ & $0.0136(6)$ \\
\hline H3 & 0.676166 & 0.416091 & 0.420511 & $0.016^{*}$ \\
\hline $\mathrm{C} 4$ & $0.71816(16)$ & 0.45269 (15) & $0.6492(5)$ & $0.0129(7)$ \\
\hline $\mathrm{C} 5$ & & 0.55309 (17) & $0.3202(6)$ & $0.0163(7)$ \\
\hline C6 & $0.88150(17)$ & $0.61460(17)$ & $0.3537(6)$ & $0.0171(7)$ \\
\hline C7 & 0.93618 (19) & 0.64297 (19) & $0.2884(7)$ & $0.0230(9)$ \\
\hline $\mathrm{H} 7$ & 0.949318 & 0.623926 & 0.216837 & $0.028^{\star}$ \\
\hline $\mathrm{C} 8$ & $0.9712(2)$ & 0.69925 (19) & $0.3290(7)$ & $0.0250(9)$ \\
\hline H8 & 1.008506 & 0.718208 & 0.285846 & 0.030 * \\
\hline C9 & $0.9526(2)$ & $0.72843(18)$ & $0.4319(7)$ & $0.0237(9)$ \\
\hline C10 & $0.8978(2)$ & $0.6992(2)$ & $0.4966(8)$ & $0.0289(11)$ \\
\hline $\mathrm{H} 10$ & 0.884736 & 0.718272 & 0.568217 & $0.035^{\star}$ \\
\hline C11 & $0.8620(2)$ & $0.6427(2)$ & $1(8)$ & $0.0238(10)$ \\
\hline H11 & 0.824885 & 0.623568 & 0.502381 & $0.029^{\star}$ \\
\hline C12 & $0.9909(2)$ & 0.790 & 0.46 & $0.0344(12)$ \\
\hline $\mathrm{H} 12 \mathrm{~A}$ & 0.978898 & 0.812356 & 0.400511 & $0.052^{\star}$ \\
\hline H12B & 1.029500 & 0.801064 & 0.438960 & $0.052^{\star}$ \\
\hline $\mathrm{H} 12 \mathrm{C}$ & 0.988853 & 0.797431 & 0.592500 & $0.052^{\star}$ \\
\hline C13 & $0.63190(18)$ & $0.4757(2)$ & $0.4750(6)$ & $0.0196(8)$ \\
\hline C14 & $0.6127(2)$ & $0.5142(2)$ & $0.4117(7)$ & 0.0257 (9) \\
\hline C15 & $0.6493(2)$ & $0.5645(2)$ & $0.3285(8)$ & $0.0300(11)$ \\
\hline H15 & 0.687625 & 0.575245 & 0.314185 & $0.036^{\star}$ \\
\hline C16 & $0.6295(3)$ & $0.5990(3)$ & $0.2666(10)$ & $0.0393(14)$ \\
\hline H16 & 0.654810 & 0.633295 & 0.211321 & $0.047^{*}$ \\
\hline C17 & $0.5736(3)$ & $0.5841(3)$ & $0.2844(10)$ & $0.0436(16)$ \\
\hline C18 & $0.5380(3)$ & $0.5346(4)$ & $0.3688(13)$ & $0.054(2)$ \\
\hline H18 & 0.499884 & & 0.385021 & $0.064^{*}$ \\
\hline C19 & $0.5562(3)$ & $0.4989(3)$ & $0.4307(10)$ & $0.0449(17)$ \\
\hline H19 & 0.530552 & 0.464553 & 0.485253 & $0.054^{\star}$ \\
\hline $\mathrm{C} 20$ & $0.5524(4)$ & $0.6213(4)$ & $0.2119(14)$ & $0.064(3)$ \\
\hline $\mathrm{H} 20 \mathrm{~A}$ & 0.583977 & 0.659361 & 0.195870 & $0.095^{\star}$ \\
\hline $\mathrm{H} 20 \mathrm{~B}$ & 0.525386 & 0.622131 & 0.292657 & $0.095^{\star}$ \\
\hline $\mathrm{H} 20 \mathrm{C}$ & 0.534045 & 0.606340 & 0.100358 & $0.095^{\star}$ \\
\hline C21 & $0.8222(2)$ & $0.3919(2)$ & $0.0253(8)$ & $0.0293(11)$ \\
\hline $\mathrm{H} 21 \mathrm{~A}$ & 0.833783 & 0.428471 & 0.078056 & $0.044^{\star}$ \\
\hline $\mathrm{H} 21 \mathrm{~B}$ & 0.854460 & 0.393081 & -0.032546 & $0.044^{\star}$ \\
\hline $\mathrm{H} 21 \mathrm{C}$ & 0.808206 & 0.362994 & 0.115298 & $0.044^{\star}$ \\
\hline
\end{tabular}

name: 2,3-bis((4-methylbenzoyl)oxy)succinic acid; $69.5 \mathrm{mg}$, $0.18 \mathrm{mmol}$ ) was dissolved in $2.5 \mathrm{~mL}$ methanol, and added to a $25 \mathrm{~mL}$ round bottom flask. An aqueous solution $(2.50 \mathrm{~mL})$ of $\mathrm{La}\left(\mathrm{NO}_{3}\right) \cdot 6 \mathrm{H}_{2} \mathrm{O}(51.9 \mathrm{mg}, 0.12 \mathrm{mmol})$ was added slowly to the solution. Then a $\mathrm{KOH}$ solution $(0.2 \mathrm{~mol} / \mathrm{L})$ was carefully added to the mixture and adjust the $\mathrm{pH}$ value of the solution to about 6.5. After stirring for $7 \mathrm{~h}$ at room temperature, the filtrate was kept undisturbed for seven days. Colorless block crystals were obtained in a yield of $26 \%$, based on D- $\mathrm{H}_{2}$ DTTA.

\section{Experimental details}

Single crystal X-ray diffraction data of the complex were collected on beam line 3W1A of the Beijing Synchrotron Radiation Facility (BSRF), using A MARCCD-165 detector $(\lambda=0.71000 \mathrm{~A})$ at a working voltage of $2.5 \mathrm{GeV}$, and the single crystals with regular shape, suitable size, and bright without cracks were selected under the polarization stereoscopic microscope. Data were collected by the MARCCD diffractometer and processed using HKL 2000 [1]. Multi-scan program SADABS was used for absorption correction [2]. The structures was solved by direct methods and refined by the full-matrix least squares on $F^{2}$ using the SHELXS-97 [3] and SHELXL-2014/7 [4]. Coordinates of hydrogen atoms were refined without any constraints or restraints. Their $U_{\text {iso }}$ values were set to $1.2 U_{e q}$ of the parent atoms.

\section{Comment}

In the past few decades, the design and application of lanthanide coordination polymers (Ln-CPs) have attracted extensive attention [5], not only the diversity of their structures, but also their wide applications in catalysis, magnetism and fluorescence detection [6]. More importantly, $\operatorname{Ln}(\mathrm{III})$ ions often endow the CPs with unique luminescent properties (characteristic sharp emission, high color purity, and long excited-state luminescence lifetimes) as a result of transitions within the partially-filled $4 f$ shells of the trivalent $\operatorname{Ln}(\mathrm{III})$ ions [7]. Compared with other metal ions, Ln(III) ions have the characteristics of variable high coordination number, strong spin orbit coupling, and large magnetic moment, and have important functions such as magnetic, fluorescence sensing, nuclear magnetic resonance imaging [8], so Ln-CPs are the potential material for environmental or biomedical applications [9]. However, Ln-CPs are sensitive to temperature, $\mathrm{pH}$, and 
pressure during synthetic process, which makes it difficult to grow crystals [10].

Rational selection of ligands is considered to be a very suitable method for the synthesis of structurally diverse Ln-CPs [11]. Tartaric acid, which is relatively inexpensive and readily available, plays an important role in many areas of chemistry [12]. D- $\mathrm{H}_{2}$ DTTA ((+)-di-p-toluoyl-d-tartaric acid) is a flexible dicarboxylic acid with two equal chiral carbon atoms. Abundant carboxyl groups can provide variable coordination modes, which contribute to the construction of metal-organic complexes. In recent years, many $\mathrm{Ln}$-CPs based on D- $\mathrm{H}_{2}$ DTTA have been reported. Zhang Yuxiao et al. reported two novel isostructural Ln-CPs $\left[\operatorname{Ln}(\mathrm{DTTA})(\mathrm{DMF})_{3}\right] \mathrm{NO}_{3}(\mathrm{Ln}=\mathrm{Eu}, \mathrm{Tb})$ [13]. Our group synthesized a series of Ln-CPs, $\left[\operatorname{Ln}(\mathrm{HDTTA})_{3}\left(\mathrm{CH}_{3} \mathrm{OH}\right)_{3}\right]_{\mathrm{n}}$ ( $\mathrm{Ln}=\mathrm{Ce}, \mathrm{Pr}, \mathrm{Sm}, \mathrm{Eu}, \mathrm{Gd}, \mathrm{Tb}, \mathrm{Dy}, \mathrm{Ho})$, and studied their optical and magnetic properties [14-17].

$\mathrm{X}$-ray single crystal diffraction results show that the title complex belongs to the trigonal crystal system with the space group R3. The asymmetric unit consists of one third of a $\mathrm{La}^{3+}$, a HDTTA ${ }^{-}$ion and a coordinated methanol molecule. $\mathrm{La}^{3+}$ is a nine-coordinated cation, and the coordinated atoms are respectively from six carboxyl oxygen atoms of $\mathrm{HDTTA}^{-}$and three oxygen atoms of methanol molecules, presenting the coordination pattern of three-capped triangular prism. The bond length of La-O ranges from 2.441(3) to 2.694(3) $\AA$, and the bond angle of $\mathrm{O}-\mathrm{La}-\mathrm{O}$ ranges from 81.15(12) to $132.64(10)^{\circ}$. The complex forms an infinite onedimensional chain structure along the $c$-axis by bridging $\mathrm{La}^{3+}$ with carboxyl groups at both ends of the ligand.

Author contributions: All the authors have accepted responsibility for the entire content of this submitted manuscript and approved submission.

Research funding: This work was supported by the Natural Science Foundation of China (Grant No 21671124) and the Scientific Instrument Center of Shanxi University of China. Conflict of interest statement: The authors declare no conflicts of interest regarding this article.

\section{References}

1. Otwinowski Z., MinorW. Processing of X-ray diffraction data collected in oscillation mode. Methods Enzymol. 1997, 276, 307-326.

2. Bruker. SMART, SAINT and SADABS; Bruker AXS Inc.: Madison, Wisconsin, USA, 1998.
3. Sheldrick G. M. A short history of SHELX. Acta Crystallogr. 2008, A64, 112-122.

4. Sheldrick G. M. Crystal structure refinement with SHELXL. Acta Crystallogr. 2015, C71, 3-8.

5. Horrock W. D., Wong C.-P. Lanthanide porphyrin complexes. evaluation of nuclear magnetic resonance dipolar probe and shift reagent capabilities. J. Am. Chem. Soc. 1976, 98, 7157-7162.

6. Zhao Z.-P., Zheng K., Li H.-R. Structure variation and luminescence of 3D, 2D and $1 \mathrm{D}$ lanthanide coordination polymers with 1,3-adamantanediacetic acid. Inorg. Chim. Acta. 2018, 482, 340-346.

7. Qiao C.-F., Zhao Y., Ren Y.-L., Gao X., Zhang M.-L., Zhou C.-S., Zhang G.-C. Synthesis, photoluminescence and thermodynamics of two lanthanide coordination polymers with 5-nitroisophthalate. J. Solid State Chem. 2019, 270, 443-449.

8. Borovkov V. Book review of "Lanthanide metal-organic frameworks". Front. Chem. 2015, 3, 50.

9. Liu Y., Xie X.-D., Xu C., Jiang H.-E., Liu W.-S. 2D Near-infrared luminescence Ln-coordination-polymers as an assistor for biomedicine. ChemistrySelect 2020, 5, 10771-10774.

10. Zhang S.-W., Duan E.-Y., Han Z.-S., Li L.-L., Cheng P. Lanthanide coordination polymers with 4,4'-azobenzoic acid: enhanced stability and magnetocaloric effect by removing guest solvents. Inorg. Chem. 2015, 54, 6498-6503.

11. Du J.-Q., Dong J.-L., Xie F., Han H.-M., Yang R.-X., Wang D.-Z. Two types of lanthanide coordination polymers showing luminescence and magnetic properties. Polyhedron 2019, 167, 26-32.

12. Bernas U., Hajmowicz H., Synoradzki L. Tartaric acid and its O-acyl derivatives. Part 14: nucleophilic ring-opening reaction of nonsymmetrically substituted tartaric acid anhydride as a tool for the synthesis of totally differentiated tartaric acid derivatives. Tetrahedron 2015, 71, 4047-4052.

13. Zhang Y.-X., Wu L., Feng M., Wang D.-M., Li C.-X. Assembly of twodimension LMOF materials with excellent detection of $\mathrm{Fe}^{3+}$ ion in water based on overlap mechanism. J. Solid State Chem. 2021, 294, 121868.

14. Han M.-R., Li S.-D., Ma L., Yao B., Feng S.-S., Zhu M.-L. Luminescent and magnetic bifunctional coordination complex based on a chiral tartaric acid derivative and europium. Acta Crystallogr. 2019, C75, 1220-1227.

15. Zhang H.-T., Ma L., Han M.-R., Feng S.-S., Zhu M.-L. A onedimensional chiral gadolinium complex based on a tartaric acid derivative: crystal structure, thermal behavior and magnetic properties. Inorg. Nano-Metal Chem. 2021, 51, 761-765.

16. Han M.-R., Zhang H.-T., Wang J.-N., Feng S.-S., Lu L.-P. Three chiral one-dimensional lanthanide-ditoluoyl-tartrate bifunctional polymers exhibiting luminescence and magnetic behaviors. RSC Adv. 2019, 55, 32288-32295.

17. Gao X.-L., Han M.-R., Ren H.-F., Feng S.-S. Synthesis, structures, luminescence and photocatalytic properties of three lanthanide complexes based on ditoluoyl-tartrate. Chin. J. Inorg. Chem. 2021, 37, 375-384. 